\title{
Maternal high-fat diet impairs glucose metabolism, $\beta$-cell function and proliferation in the second generation of offspring rats
}

\author{
Yan-Hong Huang ${ }^{1 \dagger}$, Ting-Ting Ye ${ }^{1 \dagger}$, Chong-Xiao Liu', Lei Wang ${ }^{3}$, Yuan-Wen Chen ${ }^{4}$ and Yan Dong ${ }^{1,2^{*}}$
}

\begin{abstract}
Background: This study aimed to assess the impact of perinatal high-fat (HF) diet in female Sprague-Dawley rats (F0) on glucose metabolism and islet function in their early life of second-generation of offspring (F2).

Methods: FO rats were fed with a standard chow (SC) or HF diet for 8 weeks before mating, up to termination of lactation for their first-generation of offspring (F1-SC and F1-HF). F1 females were mated with normal males at the age of week 11, and producing F2 offspring (F2-SC, F2-HF). All the offspring were fed SC diet after weaning for 3 weeks. The glucose level and islet function of F2 offspring were assessed at the age of week 3 and 12 .

Results: The F2-HF offspring had a high birth weight and maintained a higher body mass at the age of week 3 and 12, along with an impaired glucose tolerance and lower serum insulin levels compared with the F2-SC. $\beta$-cell proliferation was also impaired in the islets of F2-HF rats at the age of week 3 and 12. The pancreatic and duodenal homeobox factor-1 (Pdx1) and Neurogenic differentiation 1 (NeuroD1) expressions were decreased in the islet of F2-HF rats at the age of week 12.

Conclusions: Maternal HF diet during pre-gestation, gestation, and lactation in rats could result in the increased body weight and glucose intolerance in their early life of F2 offspring due to impaired $\beta$-cell function and proliferation.
\end{abstract}

Keywords: Maternal high-fat diet, Glucose tolerance, Islet function, Weight gain, Transgenerational effect

\section{Background}

Progressively increasing incidences of obesity and diabetes become a severe public health issue globally. It was estimated about $40 \%$ adult women overweight and $15 \%$ obese in 2014. Approximately, 42 million children under the age of 5 were also classified as overweight or obese [1]. Decreased physical activity and increased caloric intake are thought to be the key factors in the development of obesity. More recent studies in rats model, however, demonstrated that maternal high-fat diet

\footnotetext{
* Correspondence: dongyansh@sjtu.edu.cn

${ }^{\dagger}$ Equal contributors

'Department of Endocrinology, Xinhua Hospital, School of Medicine, Shanghai Jiao Tong University, 1665 Kongjiang Road, Shanghai 200092, China

${ }^{2}$ Shanghai Key Laboratory of Pediatric Gastroenterology and Nutrition, Shanghai Institute for Pediatric Research, Shanghai, China

Full list of author information is available at the end of the article
}

during gestation and lactation became a significant risk factor for metabolic abnormalities, such as obesity, impaired glucose tolerance, dyslipidemia, and early-onset nonalcoholic fatty liver disease at their early life of $\mathrm{F} 1$ offspring, even while offspring were fed with SC after weaning [2-5].

Previous studies from animal models have shown that maternal undernutrition and intrauterine growth restriction induced a glucose tolerance impairment and obesity in both F1 and F2 offspring [6-8], suggesting that the maternal undernutrition might be a genomic-free transmission across generations via a suboptimal uterine environment, which could result in programmed alterations in organ structure and function. An inadequate diet during F0 pregnancy could affect the intranterine development of the F1 offspring, which might in turn impact the F2 offspring $[6,8]$. 
There is, however, little known regarding the impact of perinatal HF diet exposure on the pancreatic islet function of subsequent generations, i.e. their offspring. Therefore, we aimed to assess the impact of a HF diet during pregestation, gestation and lactation on the glucose metabolism and $\beta$-cell function and proliferation in their early life of F2 offspring in rats.

\section{Methods}

\section{Diet on animal model}

The study protocol was approved by the Animal Use and Care Committee at our institute. Female Sprague-Dawley rats (age of 3 weeks, Shanghai SLRC laboratory animal CO. LTD) were housed in ventilated isolator cages with 12/12 light/dark cycles and a constant temperature of 22 $\pm 2^{\circ} \mathrm{C}$. Animals were given free access to water and food. The dams were fed with SC diet or HF diet for 8 weeks, and were grouped to two, F0-SC, F0-HF, respectively. The HF diet contained (kcal \%): fat, 45\%; carbohydrate, 29.5\%; and protein, $25.5 \%$ and the energy density was $4.7 \mathrm{kcal} / \mathrm{g}$, whereas the SC diet was containing (kcal \%): fat, 12.9\%; carbohydrate, $61.6 \%$; and protein, $25.5 \%$. and the energy density was $3.5 \mathrm{kcal} / \mathrm{g}$, as described previously [9]. Vitamin and mineral contents of both diets were identical (Calcium, 1.24\%; Phosphorus, 0.83\%; Lysine 1.38\%; Methionine and cystine, $0.72 \%$ ). The females from different litters were mated with males. The dams maintained their respective diets until the end of lactation after pregnancy. Five F1 female offspring in the F1-SC and F1-HF groups were selected and weaned to the $\mathrm{SC}$ diet at the postnatal day of 21 . Five F1 female offspring from different litters were mated with normal males after 8-weeks to produce F2 offspring. F1 females were fed with the SC diet throughout the pregnancy and the lactation. Six rat pups in F2-SC and F2-HF were selected (1:1 sex ratio), and fed with the SC diet up to 12-weeks after being weaned at 3weeks. The feeding flow chart was presented in Fig. 1. Note that only F0-HF dams were fed with the HF diet during pregestation, gestation and lactation. The body masses of F0 and F1 dams were measured weekly, and the birth weights of their F2 offspring were documented as well.

\section{Glucose tolerance tests (GTT)}

GTT was performed in F0 and F1 generation before gestation ( $n=5$ /group). It was also determined in two F2 offspring groups at weeks 3 and 12 ( $n=6$ /group). The rats were fasted overnight and injected intraperitoneally with $1.5 \mathrm{~g} / \mathrm{kg}$ body weight of glucose. Blood was collected from the tail vein at $0,30,60,120$ min following the glucose loading. Blood glucose levels were determined by using a handheld glucometer (One Touch Ultra, Johnson \&Johnson, New Brunswick, NJ, USA). The glucose tolerence was assessed by calculating the area under the curve (AUC) based on the trapezoid rule (GraphPad Prism version 6.0, La Jolla, CA, USA).

\section{Blood collection and insulin level}

The two groups of F2 offspring were anesthetized with $1 \%$ pentobarbital sodium intraperitoneally $(40 \mathrm{mg} / \mathrm{kg})$ overnight fasting at weeks 3 and 12. Blood was collected from cardiac puncture and preserved in the tubes, centrifuged at $3000 \mathrm{rpm} 4^{\circ} \mathrm{C}$ for $15 \mathrm{~min}$ to isolate the serum. Serum insulin level was determined using enzyme-linked immunosorbent assay (ELISA) (Rat/mouse Insulin ELISA Kit, Alpco, USA). Because there were no international units available for the rats, the amounts of insulin were converted to the units for the human by using the formula: $1 \mathrm{mg}=23.1 \mathrm{U}$. HOMA $-\beta \%=20 \times[$ insulin $] /([$ glucose $]-3.5) \%$ and applied to determine $\beta$-cell function. [10].

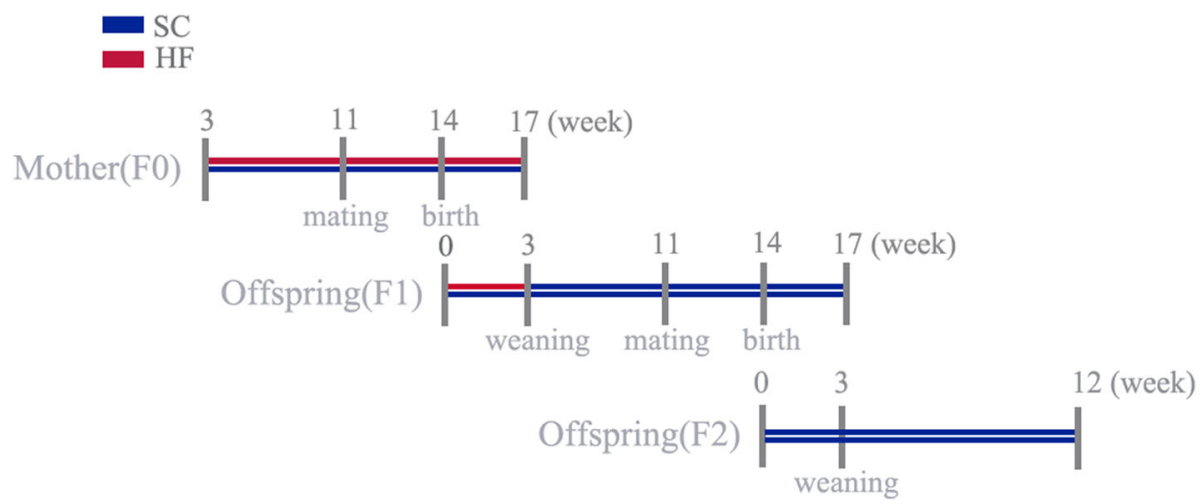

Fig. 1 The feeding flow chart for three generations. Figure 1 shows study design. Female Sprague-Dawley rats in SC group consumed a 12.9\% fat house chow diet from the age of 3 weeks and throughout the perinatal period, whereas HF group consumed a 45\% fat HF diet during the time. Both $\mathrm{F} 1$ and F2 offspring were weaned at the age of 3 weeks and fed with SC diet thereafter. The red line indicates the time period in which the dams were fed with the HF diet. The blue line presents the time period in which the SC diet was fed to the control dams, F1-generation and F2-generation offspring. HF, high-fat; SC standard chow 


\section{Immunofluorescence analysis}

The pancreas was removed and freeze-clamped immediately, and processed for immunohistostaining and mRNA and protein analyses at the age of week 3 and 12 ( $n=3$ /group). The pancreatic tissues ( $n=3$ /group) were fixed in $10 \%$ paraformaldehyde solution and sliced $3 \mu \mathrm{m}$ in thickness and assessed for immunofluorescence analysis, including immunofluorescence density using insulin (CST, USA) or Ki-67 antibodies (BD, USA) and FITC goat anti-rabbit or Alexa Fluor 594 goat antimouse IgG (Jackson, USA). 4,6-diamino-2-phenyl indole (DAPI) (Boster, China) was used to stain the nucleus. Fluorescence was visualized using the microscope (Leica DMI3000B, Germany).

Pancreatic mRNA genes, Ins1、Ins2、Pdx1、NeuroD1 determination

Total RNA ( $n=3$ /group) was extracted from the pancreas at the age of 12 -weeks ( $n=3$ /group), using Trizol reagent (Takara, Japan) according to the manufacturer's protocol, and RNA was converted into cDNA by the Power cDNA Synthesis kit (Takara, Japan). Quantitative real-time PCR was performed using ABI7500 system (ABI, Foster City, CA, USA). The mRNA for Insulin 1 (Ins1), Insulin 2 (Ins2), Pdx1, and NeuroD1 in pancreatic tissue were determined. The relative mRNA levels of qRT-PCR products were calculated by normalizing to the level of housekeeping gene $18 \mathrm{~s}$ with the $2^{-\Delta \Delta \mathrm{Ct}}$ method [11]. Gene-specific primers sequences are listed in Table 1.

\section{Western blot analyses}

The pancreatic tissue samples $(\sim 30 \mathrm{mg})$ at the age of 12 week ( $n=3$ /group) were prepared by homogenization in a Radio Immuno Precipitation Assay (RIPA) buffer with protease inhibitors and centrifuged at $12,000 \mathrm{rpm}, 4^{\circ} \mathrm{C}$ for $15 \mathrm{~min}$. The protein concentrations were quantified by modified BCA protein assay (Beyotime, China). $100 \mu \mathrm{g}$ cell lysates from each sample were separated by $12.5 \%$ SDS-PAGE gel and transferred to polyvinylidene fluoride membranes. The membranes were blocked with $5 \%$ nonfat dried milk powder in TBST buffer for $2.5 \mathrm{~h}$

Table 1 Primer sequences used for mRNA quantification by qRT-PCR

\begin{tabular}{lll}
\hline Gene & Primer sequences (5' to $3{ }^{\prime}$ ) & \\
\cline { 2 - 3 } & Forward & Reverse \\
\hline Ins1 & GTCAAACAGCACCTTTGTGG & CCAGTTGGTAGAGGGAGCAG \\
Ins2 & GTCAAACAGCACCTTTGGG & TCCAGTTGGTAGAGAGAGCAGA \\
Pdx1 & AAACCGTCGCATGAAGTGGAA & CGAGGTTACGGCACAATCCTG \\
NeuroD1 & GCAAAGGTTTGCCCAGC & ACGTGGAAGACGTGGGAG \\
$18 \mathrm{~s}$ & AAGTTCAGCACATCCTGCGAGTA & TTGGTGAGGTCAATGTCTGCTTC
\end{tabular}

Ins1 Insulin 1, Ins2 Insulin 2, $P d x 1$ pancreatic and duodenal homeobox factor-1, NeuroD1 Neurogenic differentiation 1 and were incubated with anti-Pdx1 (Abcam, USA) (1:500), anti-NeuroD1 (CST, Germany) (1:1000) and antipNeuroD1 (CST, Germany) (1:1000) overnight at $4^{\circ} \mathrm{C}$. Anti- $\beta$-actin (Sigma, USA) (1:1000) was used as an internal control. The membranes were developed using ECL reagents (Millipore, Germany) and proteins were finally detected by a chemiluminescence detection system.

\section{Statistical analyses}

Data were expressed as mean $\pm \mathrm{SE}$, and statistical analyses were performed using SPSS (version 19.0, IBM, Chicago, IL). Significant differences between $\mathrm{SC}$ and $\mathrm{HF}$ in each generation were assessed using the independent Student's $t$ test after a Pearson Normality test. $P$ value $<0.05$ was considered as significant differences between the groups.

\section{Results}

Body mass and glucose metabolism during pregestation and gestation

F0-HF and F1-HF dams were overweight compared with their corresponding control (F0-SC and F1-SC) before mating $(P=0.123,0.051$, respectively). AUC analysis of GTT showed that both the F0-HF and the F1-HF dams had a higher AUC than the F0-SC and the F1-SC $(P<0.001,<0.05$, respectively), suggesting that the glucose tolerance was impaired pre-gestation in the F0-HF and the F1-HF dams (Table 2).

\section{Body mass in F2 generation}

There was no significant difference for the average litter number and the perinatal mortality between the F2-HF and the F2-SC groups. The offspring in the F2-HF had a higher birth mass $(P<0.01)$ and body mass $(P<0.05)$, compared with the F2-SC (Table 3).

\section{Glucose metabolism in F2 generation}

Fasting blood glucose level in the F2-HF was higher than that in the F2-SC at the age of 3 weeks $(P<0.001)$. No significant difference between the F2-HF and the F2$\mathrm{SC}$ was observed in fasting blood glucose levels at the age of 12 weeks. AUC of GTT, however, was greater in the F2-HF than in the F2-SC at the age of 3,12 weeks $(P<0.001,0.05)$, indicating that the offspring in the $\mathrm{F} 2-\mathrm{HF}$ has a glucose intolerance in their early life. Moreover, the blood insulin level was lower in the F2-HF than that in the F2-SC at the age of 3,12 weeks $(P=0.031,0.013)$. The HOMA- $\beta$ index was also lower in the F2-HF than that in the F2-SC $(P=0.013,0.035$; Table 4$)$.

\section{$\beta$-cell proliferation in F2 generation}

An immunofluorescence staining of pancreatic slices was applied to determine $\beta$-cell proliferation frequency by marking up the ki67 positive $\beta$-cell/total $\beta$-cell ratio. 
Table 2 Body mass and glucose metabolism during the pregestation and the gestation in dams

\begin{tabular}{lllllll}
\hline Data & F0-SC & F0-HF & $p$-value & F1-SC & F1-HF & p-value \\
\hline$N$ & 5 & 5 & & 5 & $219.9 \pm 16.1$ & $246.3 \pm 20.2$ \\
Pregestational BM (g) & $231.7 \pm 19.2$ & $248.8 \pm 08.7$ & 0.123 & 0.051 \\
Pregestational FBG (mmol/L) & $4.36 \pm 0.49$ & $5.80 \pm 0.52^{* *}$ & 0.002 & $5.22 \pm 0.58$ & $5.08 \pm 0.26$ & 0.660 \\
Pregestational GTT-AUC (\%) & $25.3 \pm 2.1$ & $33.3 \pm 1.3^{* *}$ & $<0.001$ & $34.6 \pm 2.8$ & $40.8 \pm 4.6^{*}$ & 0.032 \\
Gestational BM (g) & $371.3 \pm 23.3$ & $396.5 \pm 24.7$ & 0.127 & $369.6 \pm 40.5$ & $399.7 \pm 21.6$ & 0.182 \\
\hline
\end{tabular}

BM body mass, FBG fasting blood glucose, GTT-AUC area under the curve of glucose tolerance, FO-SC standard-chow fed dams, FO-HF high-fat fed dams, F1-SC first generation born from standard-chow fed dam, F1-HF first generation born from high-fat fed dam

${ }^{* *}, P<0.01$ versus FO-SC

The ratio in the F2-HF rats was found to be significantly decreased as compared to the ratio in the control at the age of 3,12 weeks $(P=0.034,0.036)$, suggesting that a decreased islet $\beta$-cell proliferation in the early life of the F2-HF rats was presented (Fig. 2).

\section{Gene expression in F2 pancreas}

To further understand the mechanisms on a maternal HF diet decreasing islet function in the F2-HF, the insulin mRNA, protein, and other important transcription factors involved in the differentiation and development of $\beta$-cells were assessed at the age of 12 weeks. The mRNA levels of the Pdx1 and the NeuroD1 were found to be decreased significantly in the F2-HF with respect to that in the F2SC $(P=0.001,0.011$; Fig. 3). Consequently, Pdx1 protein expression was decreased in the F2-HF as compared with that in the F2-SC $(1.25 \pm 0.33$ versus $0.56 \pm 0.21, P=0.036$; Fig. 4); NeuroD1 protein expression was slightly reduced in the F2-HF $(0.68 \pm 0.13$ versus $0.56 \pm 0.09, P=0.278$; Fig. 4). Furthermore, an active form of NeuroD1 in pancreas, phospho-NeuroD1 (p-NeuroD1) protein expression was decreased significantly in the F2-HF as compared with that in the F2-SC $(0.66 \pm 0.20$ versus $0.31 \pm 0.04, P=0.040$; Fig. 4$)$. There were not significantly differences for the mRNA levels of the Ins1 and the Ins2 gene between the F2-HF and the F2-SC $(P>0.05$, Fig. 3$)$.

\section{Discussion}

Major findings in this study demonstrated that the trans-generational impact of F0 maternal HF diet on the F2 generation such that the F2 offspring had an

Table 3 Body mass in F2 generation offspring rats (g)

\begin{tabular}{llll}
\hline Age & F2-SC & F2-HF & $P$ Value \\
\hline N & 6 & 6 & \\
PND 1 & $5.8 \pm 0.2$ & $6.2 \pm 0.2^{* *}$ & 0.008 \\
3-week-old & $43.9 \pm 5.3$ & $55.7 \pm 9.3^{*}$ & 0.023 \\
12-week-old & $328.8 \pm 47.9$ & $391.6 \pm 34.4^{*}$ & 0.026
\end{tabular}

PND postnatal day, F2-SC second generation of grandmother fed with standard-chow diet, F2-HF second generation of grandmother fed with high-fat diet

${ }^{*}, P<0.05$ versus $F 2-S C,{ }^{* *}, P<0.01$ versus $F 2$-SC increased body weight, glucose intolerance, $\beta$-cell function and proliferation impairment, along with a decreased expression of Pdx1 and p-NeuroD1, despite being fed with the SC diet after weaning.

HF diet during the gestation and the lactation can increase body weight and make metabolic abnormalities. A growing number of animal studies recently demonstrated that HF diet during the gestation and the lactation could induce weight gain and metabolic abnormalities, such as pancreatic islet dysfunction in F1 offspring $[2-5,12,13]$. Two studies from Takasaki et al. and $\mathrm{Li}$ et al. reported that a continuous HF feeding beyond one generation (derived from both F0 and F1 maternal obesity) promoted body weight increase, hepatic lipid accumulation, and hepatic steatosis in F2 generation offspring $[14,15]$. However, both F1 and F2 offspring in these studies were consecutively fed with HF diet, the impacts of F0 maternal HF diet on F2 generation could not be distinguished from the HF diet on F1 and F2 offspring. In this study, only the F0-HF dams were fed with the HF diet, F1 generation and its offspring (F2) were given the SC diet after weaning; this could be expressed as the impacts of FO maternal HF diet on F2 generation.

The metabolic disorders in the F2-HF offspring could be attributed to the effect of their F0 maternal HF diet.

Table 4 Serum glucose characteristics in F2 generation

\begin{tabular}{|c|c|c|c|c|}
\hline Time & Glucose characteristics & F2-SC & $\mathrm{F} 2-\mathrm{HF}$ & $P$ Value \\
\hline & $N$ & 6 & 6 & \\
\hline \multirow[t]{4}{*}{ 3-week-old } & FBG (mmol/L) & $4.6 \pm 0.4$ & $6.2 \pm 0.5^{* *}$ & $<0.001$ \\
\hline & GTT-AUC & $32.4 \pm 1.7$ & $41.5 \pm 3.5^{* *}$ & $<0.001$ \\
\hline & Insulin( $\mu \mathrm{U} / \mathrm{ml})$ & $5.3 \pm 0.8$ & $4.5 \pm 0.1^{*}$ & 0.031 \\
\hline & HOMA- $\beta \%$ & $108.0 \pm 47.8$ & $34.7 \pm 7.4^{*}$ & 0.013 \\
\hline \multirow[t]{4}{*}{ 12-week-old } & $\mathrm{FBG}(\mathrm{mmol} / \mathrm{L})$ & $5.3 \pm 0.6$ & $5.8 \pm 0.3$ & 0.110 \\
\hline & GTT-AUC & $37.0 \pm 1.4$ & $40.6 \pm 3.2^{*}$ & 0.039 \\
\hline & Insulin( $\mu \mathrm{U} / \mathrm{ml})$ & $12.1 \pm 4.1$ & $5.8 \pm 0.5^{*}$ & 0.013 \\
\hline & HOMA- $\beta \%$ & $146.2 \pm 81.9$ & $50.8 \pm 8.9^{*}$ & 0.035 \\
\hline
\end{tabular}

$G$ fasting blood glucose, GTT-AUC area under the curve of glucose tolerance, HOMA- $\beta$ homeostasis model assessment of insulin secretion index, F2-SC second generation of grandmother fed with standard-chow diet, F2-HF second generation of grandmother fed with high-fat diet ${ }^{*}, P<0.05$ versus $\mathrm{F} 2$-SC, ${ }^{*}, P<0.01$ versus $\mathrm{F} 2$-SC 

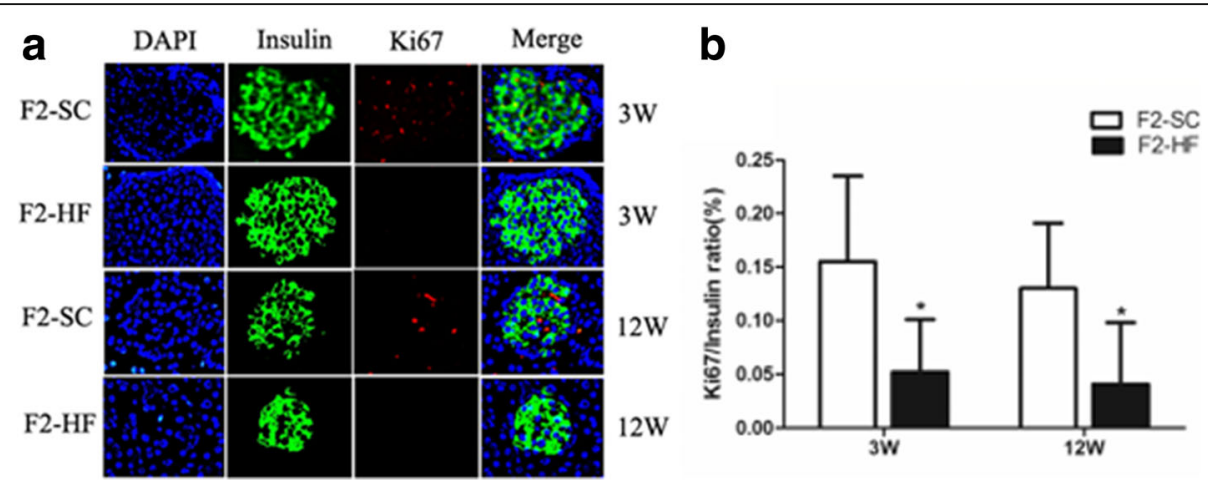

Fig. 2 Islets $\beta$-cell proliferation in F2 generation at the age of 3 and 12 weeks. a Representative immunofluorescence images of insulin and Ki67 expression; (b) Quantitative analysis of ki67 positive $\beta$-cell /total $\beta$-cell ( $\beta$-cell proliferation frequency). $\beta$-cell proliferation frequency in the F2-HF was significantly decreased compared to that in the F2-SC at the age of 3 and 12 weeks. ${ }^{*}, P<0.05$ versus F2-SC. $N=3$ per group

F1-generation from F0 fed with the HF diet in this study showed an increased body mass and an impaired glucose tolerance throughout their early life. Similarly, offspring in F2-HF also presented a higher body mass and impaired glucose tolerance, although F1 dams were fed with the SC diet after weaning and throughout the gestation and the lactation. These results implied that F0 maternal HF diet could induce a trans-generational effect on weight gain and glucose intolerance in F2 generation, similar to the trans-generation in the grandmaternal intrauterine undernutrition $[6,8]$.

It is worth mentioning that F2-HF offspring had a lower serum insulin level and a lower HOMA- $\beta$ (\%) at the ages of week 3 and week 12 in this study. This was different from the prior studies of maternal HF diet that reported a serum insulin level increase in F2-generation

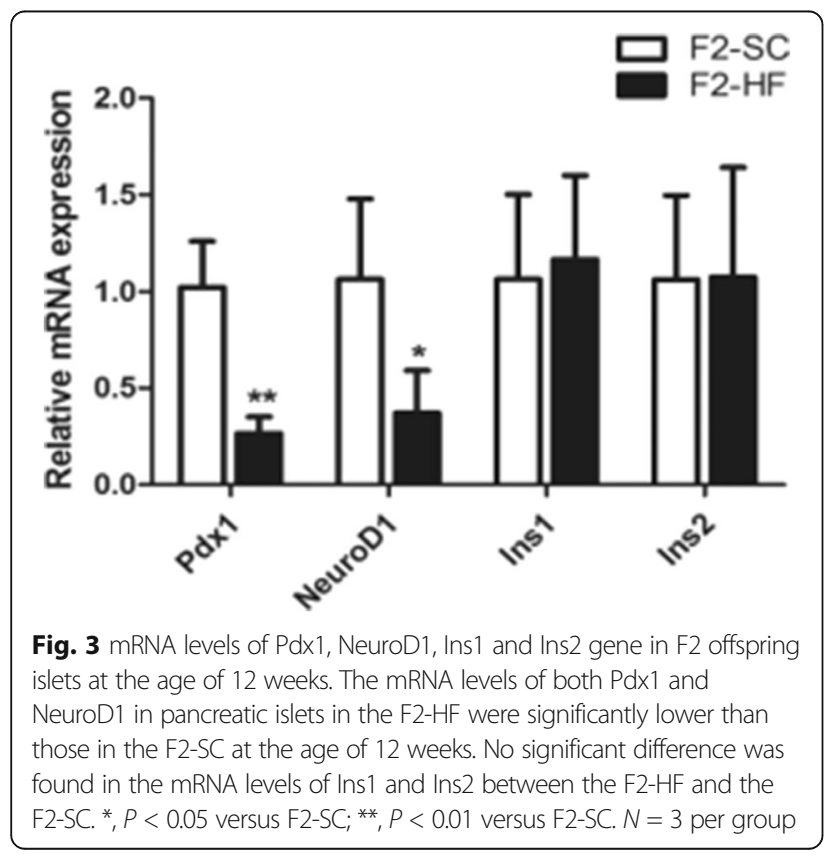

$[14,15]$. This difference could be explained by varying diets since they applied HF diet consecutively for three generations while we applied maternal HF diet only in the F0 [14, 15]. HF-feeding could also increase the risk of obesity and obesity-associated disorders, such as hyperglycemia, hyperinsulinemia, insulin resistance, and hepatic steatosis in both animals and humans $[2-5,12]$.

Understanding the mechanisms on an insulin secretion decrease in F2-HF offspring remains very important in an association of islet $\beta$-cell function to the maternal HF diet. We examined the islet $\beta$-cell proliferation frequency using the ki67 positive $\beta$-cell /total $\beta$-cell ratio in the offspring. A significant low ki67/insulin ratios at the age of 3 and 12 weeks was found in the F2-HF offspring, suggesting that F2-HF offspring had a decreased islet $\beta$-cell proliferation in their early life. Meanwhile, a corresponding decrease of Pdx1 and p-NeuroD1 expressions was observed in the pancreatic islets, which was reflected by the analytic results from the mRNA and protein expression. More interestingly, Pdx1 and NeuroD1 were pivotal transcriptional factors in the development and differentiation of pancreatic islets. These factors were critical in maintaining the islet $\beta$-cell function [16-21]. Pdx1 played a critical role in the survival of the $\beta$-cell. The decreased Pdx1 expression in pancreatic islets from type 2 diabetic patients correlated with the decreased insulin expression and decreased glucose-stimulated insulin secretion in human islets [22]. This decreased Pdx1 and p-NeuroD1 expressions in this study was closely associated with reduced $\beta$-cell mass in the F2-HF, which led to an impaired glucose tolerance in early life. As the F1-HF dams were fed with the SC diet after weaning, these findings indicated that the FO perinatal HF diet could transgenerationally impact on the $\mathrm{Pdx} 1$ and $\mathrm{p}$ NeuroD1 expression in islet, and also impact on pancreatic islet function in the second generation of offspring. 


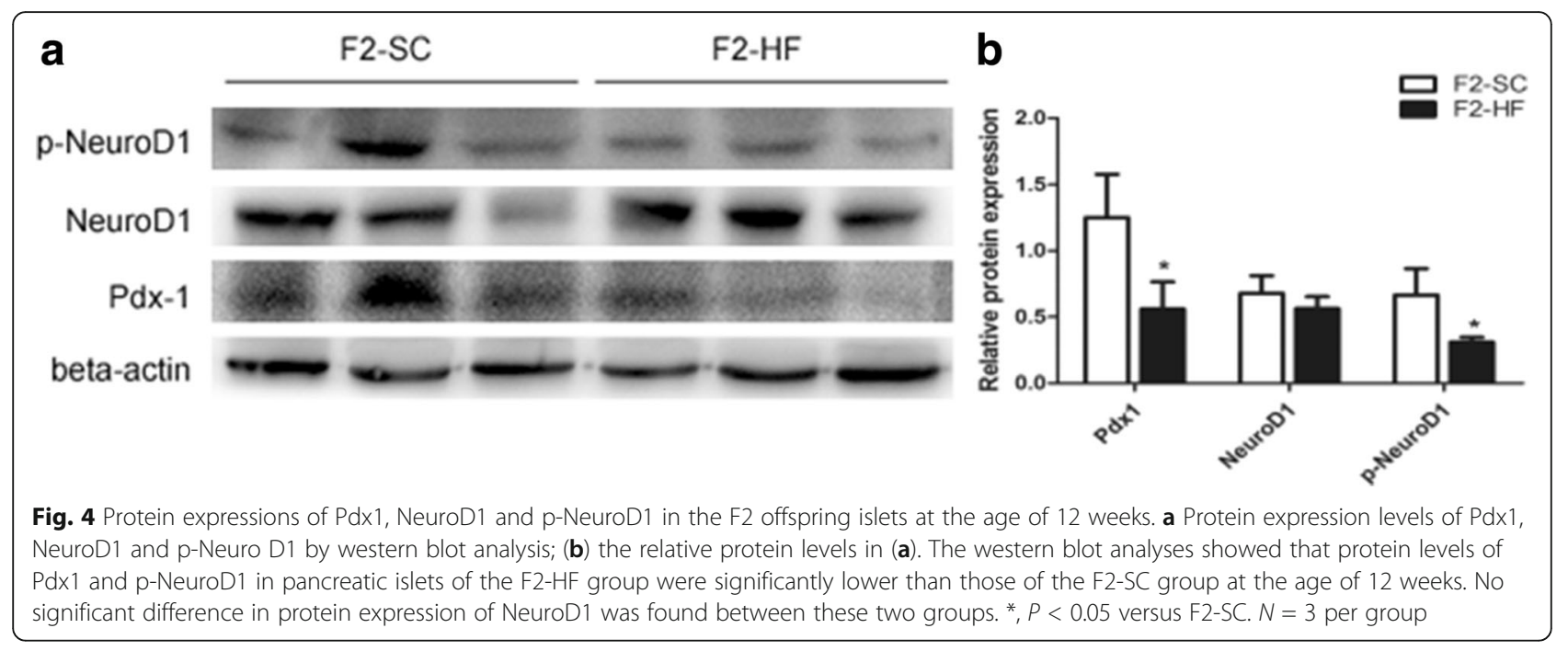

\section{Conclusion}

In summary, these findings demonstrated that the maternal perinatal HF diet could result in transgenerational abnormalities in persisting for multiple generations. These abnormalities may induce programming retardation in islet $\beta$-cell development in the early life of F2 offspring, ultimately leading to an increased body weight with impaired glucose tolerance, a reduced expression of $\mathrm{Pdx} 1$ and $\mathrm{p}-\mathrm{NeuroD} 1$, and a decreased islet $\beta$-cell proliferation in the F2 offspring.

\section{Abbreviations}

AUC: Area under the curve; DAPI: 4,6-diamino-2-phenyl indole; ELISA: Enzyme-linked immunosorbent assay; GTT: Glucose tolerance tests; HF: High-fat; HOMA- $\beta$ : Homeostasis model assessment of insulin secretion index; Ins1: Insulin 1; Ins2: Insulin 2; NeuroD1: Neurogenic differentiation 1; Pdx1: Pancreatic and duodenal homeobox factor-1; SC: Standard chow

\section{Acknowledgements}

Not applicable

\section{Funding}

This study was supported by Science Foundation of School of Medicine, Shanghai Jiao Tong University [\# 11XJ21037].

\section{Availability of data and materials}

The data that support the findings of this study are available from the corresponding author upon requesting.

\section{Authors' contributions}

$\mathrm{HYH}$ and YTT conducted the experiments and data analyses, LCX worked on a part of the experiments, WL and CYW monitored the experiment, DY monitored the experiment, the data analyses and the manuscript preparation. All authors were involved in writing this manuscript and had final approval of the submitted version.

\section{Ethics approval}

Animals were handled following the Guidelines of the China Animal Welfare Legislation, and the study protocol was approved by Ethics Committee of Xin Hua Hospital, School of Medicine, Shanghai Jiao Tong University.

\section{Consent for publication}

Not applicable

\section{Competing interests}

The authors declare that they have no competing interests.

\section{Publisher's Note}

Springer Nature remains neutral with regard to jurisdictional claims in published maps and institutional affiliations.

\section{Author details}

${ }^{1}$ Department of Endocrinology, Xinhua Hospital, School of Medicine, Shanghai Jiao Tong University, 1665 Kongjiang Road, Shanghai 200092 China. ${ }^{2}$ Shanghai Key Laboratory of Pediatric Gastroenterology and Nutrition, Shanghai Institute for Pediatric Research, Shanghai, China. ${ }^{3}$ Department of Obstetrics, Xinhua Hospital, School of Medicine, Shanghai Jiao Tong University, Shanghai, China. ${ }^{4}$ Department of Gastroenterology, Xinhua Hospital, School of Medicine, Shanghai Jiao Tong University, Shanghai, China.

Received: 14 June 2017 Accepted: 20 October 2017

Published online: 02 November 2017

\section{References}

1. Devlieger R, Benhalima K, Damm P, Van Assche A, Mathieu C, Mahmood T, et al. Maternal obesity in Europe: where do we stand and how to move forward? A scientific paper commissioned by the European board and College of Obstetrics and Gynaecology (EBCOG). Eur J Obstet Gynecol Reprod Biol. 2016;201:203-8.

2. Ohta T, Toriniwa $Y$, Ryumon N, Inaba N, Hirao T, Yamanaka S, et al. Maternal high-fat diet promotes onset of diabetes in rat offspring. Anim Sci J. 2016; https://doi.org/10.1111/asj.12606.

3. Oliveira LS, Souza LL, Souza AF, Cordeiro A, Kluck GE, Atella GC, et al. Perinatal maternal high-fat diet promotes alterations in hepatic lipid metabolism and resistance to the hypolipidemic effect of fish oil in adolescent rat offspring. Mol Nutr Food Res. 2016; https://doi.org/10.1002/ mnfr.201600171.

4. Guo F, Jen KL. High-fat feeding during pregnancy and lactation affects offspring metabolism in rats. Physiol Behav. 1995;57:681-6.

5. Dos SPG, dos Santana SL, dos Santos Cordeiro G, Matos PG, Abensur AD, Couto RD, et al. Maternal and post-weaning exposure to a high fat diet promotes visceral obesity and hepatic steatosis in adult rat. Nutr Hosp. 2015:32:1653-8.

6. Jimenez-Chillaron JC, Isganaitis E, Charalambous M, Gesta S, PentinatPelegrin T, Faucette RR, et al. Intergenerational transmission of glucose intolerance and obesity by in utero undernutrition in mice. Diabetes. 2009; 58:460-8.

7. Peixoto-Silva N, Frantz ED, Mandarin-de-Lacerda CA, Pinheiro-Mulder A. Maternal protein restriction in mice causes adverse metabolic and hypothalamic effects in the F1 and F2 generations. Br J Nutr. 2011;1(06): 1364-73. 
8. Pinheiro AR, Salvucci ID, Aguila MB, Mandarim-de-Lacerda CA. Protein restriction during gestation and/or lactation causes adverse transgenerational effects on biometry and glucose metabolism in F1 and F2 progenies of rats. Clin Sci (Lond). 2008;114:381-92.

9. Gajda AM, Pellizzon MA, Ricci MR, UIman EA. Diet-induced metabolic syndromein rodent models. Anim Lab News. 2007;74:775-93.

10. Matthews DR, Hosker JP, Rudenski AS, Naylor BA, Treacher DF, Turner RC Homeostasis model assessment: insulin resistance and beta-cell function from fasting plasma glucose and insulin concentrations in man. Diabetologia. 1985;28:412-9.

11. Bustin SA, Benes V, Garson JA, Hellemans J, Huggett J, Kubista M, et al. The MIQE guidelines: minimum information for publication of quantitative real-time PCR experiments. Clin Chem. 2009:55:611-22.

12. Sun B, Purcell RH, Terrillion CE, Yan J, Moran TH, Tamashiro KL. Maternal high-fat diet during gestation or suckling differentially affects offspring leptin sensitivity and obesity. Diabetes. 2012;61:2833-41.

13. Blondeau $B$, Joly $B$, Perret $C$, Prince $S$, Bruneval $P$, Lelièvre-Pégorier $M$, et al. Exposure in utero to maternal diabetes leads to glucose intolerance and high blood pressure with no major effects on lipid metabolism. Diab Metab. 2011;37:245-51.

14. Takasaki M, Honma T, Yanaka M, Sato K, Shinohara N, Ito J, et al. Continuous intake of a high-fat diet beyond one generation promotes lipid accumulation in liver and white adipose tissue of female mice. J Nutr Biochem. 2012:23:640-5.

15. Li J, Huang J, Li JS, Chen H, Huang K, Zheng L. Accumulation of endoplasmic reticulum stress and lipogenesis in the liver through generational effects of high fat diets. J Hepatol. 2012;56:900-7.

16. Kaneto H, Miyatsuka T, Kawamori D, Yamamoto K, Kato K, Shiraiwa T, et al. PDX-1 and MafA play a crucial role in pancreatic $\beta$-cell differentiation and maintenance of mature $\beta$-cell function. Endocr J. 2008;55:235-52.

17. Dutta S, Gannon M, Peers B, Wright C, Bonner-Weir S, Montminy M. PDX: PBX complexes are required for normal proliferation of pancreatic cells during development. Proc Natl Acad Sci U S A. 2001;98:1065-70.

18. Gannon M, Ables ET, Crawford L, Lowe D, Offield MF, Magnuson MA, Wright CV. Pdx-1 function is specifically required in embryonic beta cells to generate appropriate numbers of endocrine cell types and maintain glucose homeostasis. Dev Boil. 2008;314:406-17.

19. Naya FJ, Huang HP, Qiu Y, Mutoh H, DeMayo FJ, Leiter AB, Tsai MJ. Diabetes, defective pancreatic morphogenesis, and abnormal enteroendocrine differentiation in BETA2/neuroD-deficient mice. Genes Dev. 1997;11:232-2334

20. Kataoka K. Multiple mechanisms and functions of maf transcription factors in the regulation of tissue-specific genes. J Biochem. 2007;141:775-81.

21. Shimoda M, Chen S, Noguchi H, Matsumoto S, Grayburn PA. Neurogenic differentiation 1 directs differentiation of cytokeratin 19-positive human pancreatic nonendocrine cells into insulin-producing cells. Transplant Proc 2010;42:2071-4.

22. Yang BT, Dayeh TA, Volkov PA, Kirkpatrick CL, Malmgren S, Jing X, et al. Increased DNA methylation and decreased expression of PDX-1 in pancreatic islets from patients with type 2 diabetes. Mol Endocrinol. 2012: 26:1203-12.

\section{Submit your next manuscript to BioMed Central and we will help you at every step:}

- We accept pre-submission inquiries

- Our selector tool helps you to find the most relevant journal

- We provide round the clock customer support

- Convenient online submission

- Thorough peer review

- Inclusion in PubMed and all major indexing services

- Maximum visibility for your research

Submit your manuscript at www.biomedcentral.com/submit 